\title{
Del juego y sus dicotomías en el despliegue de lo humano*
}

\author{
Luz Miriam Restrepo Zuluaga*
}

Recibido: 15 de diciembre de $2019 \bullet$ Aceptado: 11 de febrero de 2020

\section{Resumen}

El presente artículo es resultado de un proceso investigativo que planteó la pertinencia de considerar el juego como factor determinante del desarrollo humano, desde perspectivas individuales y sociales. Para ello, se realizó una revisión bibliográfica, desde el enfoque de la teoría crítica, sobre los conceptos centrales de la investigación: juego y desarrollo humano. Posteriormente, se realizó un análisis de aquellos puntos de encuentro presentes en ambos conceptos, entendiendo la manera en que estos se relacionan a partir del abordaje de diferentes aspectos, a saber: creatividad-reproducción; colectividad; solidaridad-competencia; libertad-subordinación.

Palabras clave: creatividad-reproducción, colectividad, libertad-subordinación.

Artículo de reflexión, con financiación propia. Especialización en Pedagogía, Universidad Pedagógica Nacional. Bogotá, Colombia.

Citar como: Restrepo, M. (2020). Del juego y sus dicotomías en el despliegue de lo humano. Revista de Investigación Cuerpo, Cultura y Movimiento, 10(1), 39-49 DoI: https://doi.org/10.15332/2422474x/5960

"* Especialista en Pedagogía. Docente de la Fundación Universitaria MonserrateUnimonserrate. Bogotá, Colombia. Correo electrónico: lmrestrepo@unimonserrate.edu.co / miriamrestrepoz@gmail.com. oRcID: https://orcid.org/0000-0002-5091-4252 


\section{Of the game and its dichotomies in the unfolding of the human}

\section{Abstract}

This article is the result of a research process that raised the relevance of considering the game as a determining factor in human development - from individual and social perspectives. For this, a bibliographic review was made, from the perspective of critical theory, on the main concepts of the research: game and human development. Subsequently, an analysis of those common aspects in both concepts was carried out, understanding the way in which these are related from the approach of aspects such as: creativity-reproduction; collectivity; solidarity-competition; freedom-subordination.

Keywords: creativity-reproduction, collectivity, freedom-subordination. 


\section{Introducción}

En el marco de la sociedad actual, cuyos intereses, necesidades y costumbres de los individuos están moldeados por el mercado, se hace imprescindible teorizar y analizar perspectivas que recobren comprensiones de lo bumano. De ahí que resulte pertinente rastrear y analizar el concepto de juego, pues dicho termino contempla desarrollos relacionados con la creatividad, colectividad y libertad. Para ello, inicialmente, se expone el juego desde la perspectiva de complejidad, complementariedad y contrariedad, para posteriormente relacionarlo con aspectos y conceptualizaciones sobre desarrollo humano.

\section{Metodología}

Para llevar a cabo el análisis planteado, se realizó una revisión documental desde el enfoque de teoría crítica, donde se indagó y se cuestionó el juego como experiencia y acontecimiento de los individuos. La naturaleza del material escrito, utilizado para la recolección de información, fue de carácter institucional y formal; por tanto, los documentos revisados fueron fuentes teóricas, políticas públicas y revistas especializadas.

\section{Resultados y discusión}

\section{Consideraciones sobre el juego}

En primer lugar, es indispensable establecer que, en esta revisión, el juego no es comprendido únicamente desde la acción misma de "jugar" — propia de la infancia-, sino que, además, se entiende como posibilidad y atributo a lo largo de la vida del ser humano, que logra resignificar la existencia de los individuos.

De esta manera, se concibe en el juego una perspectiva múltiple, diversa, contradictoria, pero, sobre todo, compleja, pues hace alusión a dicotomías que se encuentran explicitas e implícitas en la acción de jugar. Al respecto, Leif y Brunelle (1978) establecen que en el juego se experimenta "vértigo y aferramiento, simulacro y representación, azar y seguridad, competición y solidaridad [...] La regla y lo arbitrario; lo secreto y lo compartido; lo prohibido y lo autorizado; lo incierto y lo codificado; lo real y lo ficticio" (p. 39). En este sentido, se expresa en el juego diversidad de posibilidades y se lo 
considera, además, como acción corporal, social, intelectual que se materializa entre la oposición y la complementariedad.

El juego cobra un carácter equivalente a la existencia misma del ser humano, pues, ciertamente, la vida de los individuos transcurre en un sinnúmero de contrariedades que construyen su realidad. Claro ejemplo de ello se observa en la cotidianidad, con la felicidad, la incertidumbre, el fracaso, la tranquilidad, que suelen constituir episodios intermitentes de la vida. En este sentido, Debord (2013, p. 29) expresa: "El juego ha de invadir la vida entera, rompiendo radicalmente con un tiempo y un espacio lúdicos limitados". Por tanto, establece que se debe superar la separación entre juego y vida corriente.

Asimismo, resulta pertinente comprender que el juego ha de ser una acción libre y voluntaria; la libertad, entonces, se constituye en una de sus características definitorias. Caillois (1986) afirma que "el jugador no podría estar obligado sin que el juego perdiera al punto su naturaleza [...]" (p. 37).

Cabe mencionar, además, que una de las mayores particularidades y atributos del juego es la novedad. Así pues, Debord (2013) define el juego como "la experimentación permanente de novedades lúdicas" (p. 30). De esta manera, el juego favorece y propicia un continuo descubrimiento y entendimiento de aspectos y situaciones que no están bajo el dominio y comprensión total de los sujetos. Promueve la exploración, el interés y -en el mejor de los casos- la disciplina; por tanto, la experimentación del juego favorece el cambio, el enriquecimiento, la agudeza y el entendimiento del individuo y de su contexto.

Desde el panorama general anteriormente expuesto, un abordaje complejo del juego sugiere, además, un análisis de cuestiones sobre lo humano y lo animal, lo individual y lo social, y lo productivo y lo ocioso. Es indiscutible la existencia de conductas de juego en la vida animal; no obstante, el juego en los animales se reduce a la "exploración y adaptación al ambiente y, por ello, los animales cuya conducta está muy programada, como las hormigas o las abejas, no manifiestan comportamiento lúdico (Delval, 2004, p. 291)”.

Por el contrario, el juego en el ser humano suscita connotaciones mayores, gracias a la condición innata de inmadurez y plasticidad. El juego se configura como promotor de diversas posibilidades; para Bruner (1972, citado por Delval, 2004, p. 289), el juego está ligado directamente a la inmadurez con que nacen los seres humanos y que es el que le va a permitir producir una gran cantidad de conductas muy variadas que dan lugar a una adaptación muy 
flexible. Por lo tanto, el juego representa la posibilidad de cambio, transformación y despliegue de lo humano.

Por otro lado, autores como Echeverría (1980) plantean el juego desde perspectivas más amplias, y no solo desde el individuo, sino también desde lo social. Este autor afirma que la escritura, la economía, los toros y la guerra pueden ser considerados como otras maneras de jugar. Dicha postura es compartida por Huizinga (2000), quien establece la existencia de una relación directa entre juego-cultura, juego-guerra, juego-saber, juego-poesía, entre otros.

Por último, y retomando un planteamiento anteriormente mencionado, el juego no debe ser considerado una práctica exclusiva de la infancia, sino que debe manifestarse a lo largo de la existencia humana, lo cual rompe con percepciones legitimadas que establecen la infancia como etapa propicia para el juego y la adultez como etapa productiva.

\section{Consideraciones sobre el desarrollo humano}

Son varias las definiciones que en la literatura hay sobre el concepto de desarrollo humano. Sabogal (2009) afirma que el desarrollo se define como “desdoblamiento de lo que está en germen” (p. 171). Por su parte, Delval hace alusión al desarrollo como un

[...] proceso que experimenta un organismo que cambia en el tiempo hasta alcanzar un estado de equilibrio. En el caso del ser humano este nace con una serie de conductas y de disposiciones que se van a ir especificando a lo largo del tiempo. (Delval, 2004, p. 21)

Asimismo, Ferro, Amar y Abello (1998), establecen que el desarrollo humano

[...] es un proceso en el cual el ser biológico se constituye en un ser social y cultural. [...] el ser humano es el principal actor de su desarrollo, en cuyo proceso de comprensión y auto comprensión se produce una serie permanente de acciones y reacciones con otras personas en la búsqueda del perfeccionamiento de sus potencialidades. (p. 6)

En últimas, los autores concuerdan con que el desarrollo humano es un proceso multidimensional, continuo, integral y adaptativo.

Por su parte, el Programa de las Naciones Unidas para el Desarrollo lo define así: 
El desarrollo humano supone la expresión de la libertad de las personas para vivir una vida prolongada, saludable y creativa; perseguir objetivos que ellas mismas consideren valorables; y participar activamente en el desarrollo sostenible y equitativo del planeta que comparten. Las personas son beneficiarias e impulsoras del desarrollo humano, ya sea como individuo o como grupo. (Programa de las Naciones Unidas para el Desarrollo, 2010, p. 3)

En este sentido, se identifica el desarrollo humano como proceso, cambio y desdoblamiento, en el que se interrelacionan aspectos biológicos, culturales y sociales, para el perfeccionamiento de las potencialidades individuales y colectivas. Su acción se da de manera multidimensional, continua, integral y adaptativa, y toma en consideración aspectos como libertad, salud, educación, calidad de vida, existencia creativa, colectividad, sostenibilidad y desarrollo social.

No obstante, en la mayoría de definiciones, se desconoce la complejidad y contrariedad de la existencia humana, de manera que se encuentran recurrentemente discursos y posturas fraccionadas del ser humano, que no incluyen discusiones sobre deberes, realidades, conflictos, legados, debilidades, entre otros, los cuales deben ser reconocidos por planteamientos que esperan profundizar la perspectiva humana.

$\mathrm{Al}$ realizar un breve recorrido por teorizaciones sobre el juego y el desarrollo humano, se logró identificar una relación implícita entre ambas concepciones; para tal fin, se estableció un énfasis — desde la complejidad que propone la teorización sobre juego- en tres aspectos que se identifican como puntos de encuentro: creatividad-reproducción; colectividad: solidaridad y competencia; y libertad-subordinación.

\section{Jugar, crear y reproducir: posibilidades \\ del pensamiento abstracto y divergente}

Todo cuanto existe en la realidad humana ha pasado, en doble vía , por procesos producto de prácticas de reproducción y creación. Por consiguiente, al hablar sobre desarrollo humano, es necesario aludir a la capacidad que posee el ser humano de representar su realidad, siendo capaz de reproducir, imaginar, crear, innovar y transformar su existencia. La acción de crear o de reproducir implica, en el ser humano, el desarrollo de habilidades psíquicas 
que le permiten moverse en el marco cognitivo y abstracto de la realidad: "Nos hemos desarrollado más allá del mundo físico y viviente. Es en este más allá que opera el pleno desplegamiento de la humanidad" (Morin, 1999, p. 20).

La capacidad de "representar" se configura desde la infancia. El ser humano al nacer posee ciertas capacidades para actuar y relacionarse con el medio exterior, denominadas reflejos. Al ejercitar sus reflejos, el niño poco a poco logra consolidar conductas y acciones que todavía son incipientes. Repetir una y otra vez una acción refleja le permite al niño empezar a dominar su acción sobre el mundo. De esta manera, se presencian los primeros rasgos de actividad psíquica, es decir, de representación de su realidad.

Ciertamente, la capacidad de representar la realidad desde la posibilidad de "reproducir" y de "crear" se consolida gracias a la aparición del juego en los individuos, pues este favorece el desarrollo de la imaginación, la reproducción e innovación, lo que posibilita la construcción del pensamiento abstracto y divergente - cualidades propias de la especie humana y, por tanto, del desarrollo humano-. Durante los primeros años, el ser humano domina con mayor facilidad la acción de reproducir.

Vigotsky (1989) sostiene que "el pequeño comienza con una situación imaginaria que inicialmente está muy cerca de la situación real. Reproduce exactamente esta última” (p. 156). No obstante, poco a poco el ser humano experimenta la necesidad de ir más allá de la reproducción de la realidad y empiezan a desarrollarse en él habilidades de resignificación, lo que le permite crear e inventar a su voluntad: "En el juego hay un gran placer por representar la realidad vivida de acuerdo con las propias interpretaciones, y por tener el control para modificar o resignificar esa realidad según los deseos de quien juega" (Comisión Intersectorial para la Atención Integral de la Primera Infancia, 2013, p. 110).

La acción de crear, de inventar, es decir el desarrollo de la creatividad, supone en el ser humano "descubrir nuevas facetas de su imaginación, pensar en numerosas alternativas para un problema, desarrollar diferentes modos y estilos de pensamiento" (Chapouille, 2007, p. 63). Por consiguiente, se puede concluir que es el juego el posibilitador natural e inherente del desarrollo del pensamiento abstracto y divergente, que constituye la acción de jugar como factor propulsor de características consecuentes y propias del desarrollo humano. 


\section{Cooperación y competitividad: ambivalencias del carácter social del ser humano}

El ser humano dentro de sus particularidades más sobresalientes ha desarrollado, entre otras, capacidades únicas de organización, colaboración, comunicación y competición. En relación con ello, Morin (1999) afirma: "La hominización desemboca en un nuevo comienzo. El homínido se humaniza. Desde allí, el concepto de hombre tiene un doble principio: un principio biofísico y uno psico-sociocultural, ambos principios se remiten el uno al otro" (p. 20). Se reconoce, de esta manera, el carácter social del ser humano como uno de los factores determinantes de la especie humana.

El carácter social logra materializarse en la interacción; se plantea, de esta manera, dos posibilidades de dicha interacción: cooperación y competencia. Fiske (citado por Delval, 2004) establece como uno de los rasgos diferenciadores del ser humano "disponer de una gran capacidad de cooperación y competición con otros hombres” (p. 8). Morin (1999) expresa al respecto que el cerebro humano integra la agresividad y el celo (correspondiente al paleocéfalo) tanto como la afectividad (mesocéfalo). El juego no es ajeno a dichos planteamientos puesto que "es un importante instrumento de socialización y comunicación, es uno de los caminos por los cuales los niños y niñas se incorporan orgánicamente a la sociedad a la que pertenecen" (Garaigordobil, 2005, p. 39). Así pues, en el juego se expresa tanto la acción cooperativa como la acción competitiva.

La competencia supone "una atención sostenida, un entrenamiento apropiado, esfuerzos asiduos y la voluntad de vencer, implica disciplina y perseverancia” (Caillois, 1986, p. 45). En tanto, la cooperación se presenta como la posibilidad en que

[...] el cuerpo dialoga con otros cuerpos para manifestar el placer que le provocan algunas acciones, para proponer nuevas maneras de jugar y para esperar el turno, esperar lo que el otro va a hacer con su cuerpo y preparar el propio para dar respuesta a ese cuerpo que, sin lugar a duda, merece ser escuchado, interpretado, comprendido, cuestionado. Se encuentra también la capacidad de planear y organizar el juego por medio del lenguaje. (Ministerio de Educación Nacional, 2014, p. 17) 
Así pues, el juego expresa habilidades valiosas en el ser humano como: entrenamiento, esfuerzo, voluntad, disciplina, perseverancia, estrategia; es decir, competencia, pero además, favorece aspectos como la escucha, organización, integración, dialogo y convivencia —es decir, cooperación-. En este sentido, el juego resulta consecuente con el carácter social que ha posibilitado el desarrollo humano.

\section{Autocontrol, autonomía y libertad en el juego}

El ser humano logra constituirse como tal en la posibilidad de actuar de acuerdo con su voluntad, interés y deseo. De esta manera, al nacer, los individuos emprenden una búsqueda del dominio de sí. En dicha búsqueda, el juego representa un gran promotor del autocontrol y la autonomía, y se configura, de esta manera, en posibilitador del ejercicio pleno de la libertad.

A través de las primeras apariciones del juego, el ser humano logra hacer uso de su cuerpo y mente en consonancia a su voluntad. Por consiguiente, Garaigordobil (2005, p. 37) afirma que, en el juego, el ser humano coordina los movimientos de su cuerpo, que se tornan progresivamente más precisos y eficaces; desarrolla su capacidad perceptiva (percepción viso espacial, auditiva, rítmico-temporal); y conquista su cuerpo y el mundo exterior.

Con el transcurrir del tiempo, el juego se torna social y adquiere, en alguna de sus manifestaciones, una característica posibilitadora del autocontrol: la regla. Durante el juego el ser humano debe adoptar voluntariamente una serie de comportamientos y acciones propias de la situación de juego, lo cual exige en él dominio de sí, autodelimitación, autodeterminación y autorregulación. En este sentido, Vigotsky (1989) afirma que "el mayor autocontrol del que es capaz un niño se produce en el juego" (p. 151).

En ese proceso de construcción de autocontrol, dominio de sí, acción consecuente con la voluntad propia, se configura paralelamente el desarrollo de la autonomía en el ser humano, pues posibilita de manera constante la toma de decisiones y el reconocimiento de sus deseos e intereses.

De esta manera, el juego se configura como ejercicio pleno de la libertad. Se constituye, así, la libertad como característica esencial del juego. Huizinga (2000) afirma que "el juego, en su aspecto formal, es una acción libre [...] (p. 27)". Por su parte, Caillois (1986) establece "no cabe duda de que el juego se debe definir como una actividad libre y voluntaria” (p. 31). 
Por tanto, resulta contradictorio pensar que algún individuo sea obligado a jugar sin considerar que, dicha contradicción atenta a la lógica propia del juego.

Directamente relacionada con esta característica esencial del juego, se encuentra el placer. Al jugar, el ser humano experimenta el placer en múltiples formas:

El juego es una actividad placentera que genera satisfacción emocional. Es una fuente de placer y procura placer de muy distintas naturalezas: placer de crear, de ser causa y provocar efectos, placer de hacer lo prohibido, placer por el movimiento, placer de destruir sin culpa. (Garaigordobil, 2005, p. 40)

$\mathrm{Al}$ respecto, Delval (2004) afirma: "Lo que sí parece evidente en todas las actividades de juego es que los que las realizan encuentran un placer claro en ejecutarlas y que lo hacen por la satisfacción que les produce" (p. 284). Así pues, se experimenta en el juego satisfacción intima de exponerse ante situaciones creadas de manera voluntaria.

\section{Conclusiones}

Tras esta revisión bibliográfica, se afirma que el juego debe transitar por toda la existencia humana, pero que es deber de la sociedad entender la verdadera naturaleza del juego y del ser humano. En este sentido, es necesario identificar y recuperar el juego en el transcurso de la vida, puesto que este se transforma, y adquiere, renueva, crea y retoma diversos elementos que, claramente, dependen de la experiencia de vida de cada individuo.

De esta manera, el juego en la primera infancia, en la adolescencia o en la vejez - en el marco del desarrollo humano- será construido con diferentes elementos, pero indiscutiblemente deberá girar en torno a situaciones de complejidad, novedad, voluntad, creatividad-reproducción, solidaridadcompetencia, libertad-subordinación.

Asimismo, el juego, desde la perspectiva de creatividad y reproducción, es consecuente con el desarrollo humano al favorecer el pensamiento divergente y abstracto, y establece la manera en que dichas posibilidades del pensamiento favorecen el desarrollo de la especie humana y la sociedad. 
Por último, el juego posibilita acciones colectivas desde la solidaridad o la competencia, propias de la naturaleza humana, en la necesidad de disciplinar, agudizar o especializar habilidades, capacidades y conocimientos. En varias oportunidades, esto ha promovido a la humanidad y, en otras, la ha sometido.

\section{Referencias}

Caillois, R. (1986). Los juegos y los hombres, la máscara y el vértigo. México: Fondo de Cultura Económica.

Chapouille, M. (2007). La importancia del juego en el proceso educativo. Experiencias y Propuestas en la Construcción del Estilo Pedagógico en Diseño y Comunicación, 8(8), 62-63. https://fido.palermo.edu/servicios_dyc/publicacionesdc/vista/detalle_articulo.php?id_libro=10\&id_articulo=1388

Comisión Intersectorial para la Atención Integral de la Primera Infancia. (2013). Estrategia de atención integral a la primera infancia. De cero a siempre. Bogotá: Imprenta Nacional.

Debord, G. (2013). Filosofía para indignados. Textos situacionistas. Barcelona: RBA Libros.

Delval, J. (2004). El Desarrollo Humano (6. ${ }^{\mathrm{a}}$ ed.). Madrid: Siglo XXI.

Ferro, J., Amar, J. J. y Abello, R. (1998). Desarrollo humano. Perspectivas del siglo XXI. Barranquilla: Ediciones Uninorte.

Garaigordobil, M. (2005). Importancia del juego infantil en el desarrollo humano. Aula De Infantil, 25, 37-43. Recuperado de http://www.sc.ehu.es/ptwgalam/ art_completo/Aula\%20infantil.pdf

Leif, J. y Brunelle, L. (1978). La Verdadera Naturaleza del Juego. Buenos Aires: Capelusz.

Morin, E. (1999). Reseña. Los siete saberes necesarios para la educación del futuro. Ra-Ximhai, 1(3), 653-665. DoI: https://doi.org/10.35197/rx.01.03.2005.14.ha

Programa de las Naciones Unidas para el Desarrollo. (2010). Informe sobre desarrollo humano. La verdadera riqueza de las naciones: camino al desarrollo humano. Madrid: Ediciones Mundi-Prensa.

Sabogal, J. (2009). Desarrollo humano multidimensional. Nariño: Editorial Universitaria Universidad de Nariño.

Vigotsky, L. (1989). El desarrollo de los procesos psicológicos superiores del niño. Barcelona: Crítica. 